\title{
Observer-Based Robust Control for Hydraulic Velocity Control System
}

\author{
Wei Shen, ${ }^{1}$ Jihai Jiang, ${ }^{1}$ Hamid Reza Karimi, ${ }^{2}$ and Xiaoyu Su${ }^{3}$ \\ ${ }^{1}$ School of Mechatronics, Harbin Institute of Technology, Harbin 150080, China \\ ${ }^{2}$ Faculty of Technology and Science, University of Agder, 4898 Grimstad, Norway \\ ${ }^{3}$ College of Automation, Harbin Engineering University, Harbin 150001, China
}

Correspondence should be addressed to Wei Shen; shenw@hit.edu.cn

Received 5 November 2012; Accepted 15 January 2013

Academic Editor: Engang Tian

Copyright (c) 2013 Wei Shen et al. This is an open access article distributed under the Creative Commons Attribution License, which permits unrestricted use, distribution, and reproduction in any medium, provided the original work is properly cited.

\begin{abstract}
This paper investigates the problems of robust stabilization and robust control for the secondary component speed control system with parameters uncertainty and load disturbance. The aim is to enhance the control performance of hydraulic system based on Common Pressure Rail (CPR). Firstly, a mathematical model is presented to describe the hydraulic control system. Then a novel observer is proposed, and an observed-based control strategy is designed such that the closed-loop system is asymptotically stable and satisfies the disturbance attenuation level. The condition for the existence of the developed controller can by efficiently solved by using the MATLAB software. Finally, simulation results are provided to demonstrate the effectiveness of the proposed method.
\end{abstract}

\section{Introduction}

It is well known that construction machinery has been widely employed in many occasions, but the efficiency of hydraulic system which is the main transmission model is low. This is primarily why the construction machinery has the drawbacks of high oil consumption and bad emission [1]. In recent years, energy-saving research of the hydraulic system becomes hotspot due to growing energy crisis. If the element which converts the mechanical energy into hydraulic energy could be called primary element, the component which can interchange between mechanical energy and hydraulic energy (hydraulic motor/pump) would be named as secondary component $[2,3]$. The hydraulic secondary regulation system is that it can regulate the hydraulic secondary components which interconvert between mechanical energy and hydraulic energy to realize the energy transformation and transmission. Moreover, this kind of system includes energystorage components. Hence, it can recover braking energy and gravity potential energy. The secondary regulation system can be divided into Common Pressure Rail (CPR) system and flow coupled system $[4,5]$. CPR system is suit for construction machinery since it could not only recuperate energy but also keep the engine work in the high efficiency area. Therefore, this technique can reduce the oil consumption. Moreover, it can drive multiple uncorrelated loads simultaneously because the pressure is almost constant in theory. However, in practice hydraulic system always includes parameter uncertainty, nonlinearity, and load disturbance $[6,7]$. It is difficult to obtain ideal performance since the precise model is difficult to investigate and then the classical control method is not used well. In the construction machinery area especially, the good performance is difficult to obtain because the bad working condition also results in that the load fluctuates frequently and greatly. For example, the load of swing system in an excavator as the disturbance changes along with different mass of soil, and when mechanical arms work simultaneously, it will also cause the load change. The hydraulic speed control system should meet all of these requirements and it is hard for the traditional PID controller to get a good performance [8]. Energy saving and good performance hydraulic system is the development trend, especially for construction machinery. In 
recent years, the sensors are used widely in the construction machinery to pursue the good performance; they also help improve the control performance by using advanced control algorithms.

In order to suppress interference, we can adopt novel control methods such as adaptive control [9, 10], fuzzy control [11], and robust control [12-26]. Among the existing approaches, adaptive control method requires large computation and the real-time capability is poor. The precision of fuzzy control is sensitive and is dependent on high experiences. Robust control approach can convert the error between mathematical model and realistic plant into the uncertainty of the mathematical model. Robust controller can guarantee the performances of the resulting closedloop systems and satisfy all admissible uncertainties. In [14], the author deals with the problem of robust control for delayed singular systems with parametric uncertainties. $H_{\infty}$ control of uncertain systems with time delay in state or input is investigated in $[18,19]$, and the result shows much less conservativeness. Reference [25] is concerned with the problem of robust output feedback control for a class of uncertain discrete time delayed nonlinear stochastic systems. However, there are few results on robust control for velocity control system to improve the systems performance. Furthermore, more sensors are required by collecting signals to obtain the higher performance, but this is improper for construction machinery because of the low reliability due to the serious working condition which is harmful for the sensors.

Motivated by the previous discussion, this paper investigates the problem of robust control for the hydraulic velocity control system with parameters uncertainty and load disturbance. First, we select the swing system of one excavator as the research object and build the mathematical model. Then, by constructing a novel proportional and derivative observer, an observer-based controller is designed such that the closed-loop system is asymptotically stable and satisfies the disturbance attenuation level. Moreover, MATLAB software is used to solve the controller according to the new method. Finally, simulation results are provided to illustrate the effectiveness of the proposed method.

\section{System Modeling}

In $\mathrm{CPR}$, the constant pressure variable pump and hydraulic accumulator constitute the high pressure oil sources, and multiple different loads connect in parallel between the high press and low press oil passage. When the load changes, the pressure of system has small fluctuation while the flow varies with the load, and the control target such as position, velocity, or power can be reached by regulating the displacement of hydraulic pump/motor. The velocity control system block diagram based on CPR is depicted in Figure 1. The current signal output to the electric-hydraulic servo valve is processed by controller, and the inclination angle of the swash plate can be changed as desired by adjusting the position of variable displacement cylinder. The aim of driving or braking the load is then achieved by the adjustment of rotational velocity. We choose the swing system of $20 t$ excavator as the model to design the controller; the detailed period is written in the following.

2.1. Servo Valve Flow Rate Equation. The linear servo valve flow equation is

$$
q_{L}=K_{q} K_{i} K_{s} u_{i}-K_{c} p
$$

where $q_{L}$ and $u_{i}$ are, respectively, cylinder flow and input control voltage, $K_{i}, K_{s}, K_{q}$, and $K_{c}$ are different kinds of gain: they are the gain of the amplifier, servo valve coefficient, servo valve flow gain, and servo valve flow-pressure coefficient, respectively, and $p$ represents cylinder pressure difference.

2.2. Continuity Equation in the Hydraulic Cylinder. Applying the continuity equation to each of the cylinder chambers and the flow rate of cylinder yields

$$
\begin{aligned}
q_{L}= & A_{g} \frac{d y}{d t}+C_{i c}\left(p_{1}-p_{2}\right)+\frac{C_{e c}}{2}\left(p_{1}-p_{2}\right) \\
& +\frac{1}{2 \beta_{e}}\left(\frac{V_{t}}{2} \frac{d p_{1}}{d t}-\frac{V_{t}}{2} \frac{d p_{2}}{d t}\right) \\
& +\frac{A_{g} y}{2 \beta_{e}}\left(\frac{d p_{1}}{d t}+\frac{d p_{2}}{d t}\right),
\end{aligned}
$$

where $A_{g}$ represents area of variables cylinder, $y$ is the displacement of variables cylinder, $\beta_{e}, C_{i c}, C_{e c}$, and $V_{t}$ are coefficients; they are the effective bulk modulus of system, internal or cross-port leakage coefficient of cylinder, external leakage coefficient of cylinder, and total volume of fluid under compression in both chambers, respectively. The volume $A_{g} y$ is usually much smaller than $V_{t}$, and $d p_{1} / d t+d p_{2} / d t \approx 0$ because $p=p_{1}-p_{2}$; thus,

$$
q_{L}=A_{g} \frac{d y}{d t}+C_{t c} p+\frac{V_{t}}{4 \beta_{e}} \frac{d p}{d t},
$$

where $C_{t c}$ is total leakage coefficient of variables cylinder.

The equilibrium of forces equation by applying Newton's second law to the forces on the cylinder yields

$$
A_{g} P=m \frac{d^{2} y}{d t^{2}}+B_{c} \frac{d y}{d t}+K y+F,
$$

where $m$ is total mass of cylinder and load referred to cylinder, $B_{c}$ represents viscous damping coefficient of cylinder $(\mathrm{N} \cdot \mathrm{s} / \mathrm{m})$, and $K$ and $F$ are load spring gradient and load disturbance, respectively.

2.3. Torque Motion Equation at the Load. The relationship between the instantaneous volume of the secondary component and displacement of the cylinder can be deduced as follows

$$
D=\frac{y}{y_{\max }} D_{\max },
$$

where $D_{\max }$ is maximum volume of the secondary component and $y_{\max }$ is maximum displacement of cylinder. 


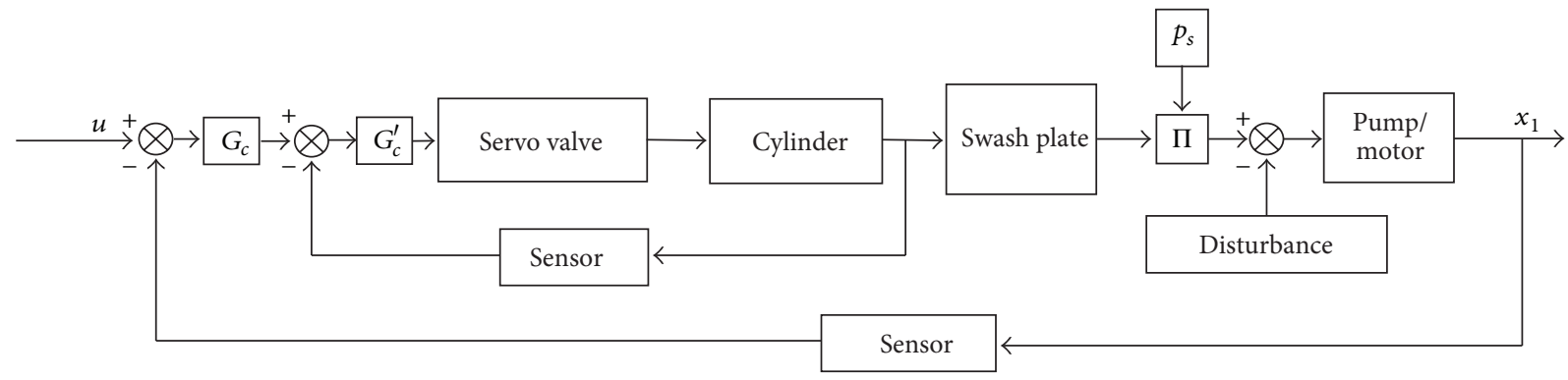

FIGURE 1: Velocity control system block diagram.

Another basic relation is the torque balance equation for the secondary component which is expressed as

$$
p_{s} \frac{y}{y_{\max }} D_{\max }=J_{p / m} \frac{d^{2} \theta}{d t^{2}}+B \frac{d \theta}{d t}+\frac{M_{f}}{r},
$$

where $\theta$ denotes angular position of the secondary component, $p_{s}$ is pressure of the high pressure pipeline in CPR, $J_{p / m}$ represents total inertia of secondary component and transformational load, $r$ is reduction ratio, and $M_{f}$ is the total including friction torque of swing system, wind resistance torque, and slope resistance torque.

2.4. System State-Space Model. State variables are selected as $X=\left[\begin{array}{llll}x_{1} & x_{2} & x_{3} & x_{4}\end{array}\right]^{T}$, where $x_{1}$ is output angular velocity of secondary component, $x_{2}$ is displacement of cylinder, $x_{3}$ is velocity of cylinder, and $x_{4}$ is pressure difference of cylinder. Thus, the state equation can be given by

$$
\begin{aligned}
& \dot{x}(t)=\left(\begin{array}{c}
-\frac{B}{J_{p / m}} x_{1}+\frac{P_{s} D_{\max }}{J_{p / m} \cdot y_{\max }} x_{2} \\
x_{3} \\
-\frac{K}{m} x_{2}-\frac{B_{c}}{m} x_{3}+\frac{A_{g}}{m} x_{4} \\
-\frac{4 \beta_{e} A_{g}}{V_{t}} x_{3}-\frac{4 \beta_{e}\left(C_{t c}+K_{c}\right)}{V_{t}} x_{4}
\end{array}\right) \\
& +\left[\begin{array}{c}
0 \\
0 \\
0 \\
\frac{4 \beta_{e}}{V_{t}} K_{i} K_{s} K_{q}
\end{array}\right] u(t) \\
& +\left[\begin{array}{c}
-\frac{M_{f}}{r \cdot J_{p / m}} \\
0 \\
-\frac{F}{m} \\
0
\end{array}\right] \text {, } \\
& y(t)=\left[\begin{array}{llll}
1 & 0 & 0 & 0
\end{array}\right]^{T} x(t) .
\end{aligned}
$$

The previous constructed model is based on ideal condition. However, the real parameters are variable due to the hard working period of excavator. We consider the parameters variation in $A$. Moreover, we add $C_{d} d(t)$ to the output because the accuracy of sensor is also influenced with temperature variation and electrical equipments. Finally, since $B_{d}$ is changing with the different load, the time varying function $f(x)$ is presented to simulate the real status. Hence, the state equations are proposed as follows:

$$
\begin{gathered}
\dot{x}(t)=(A+\Delta A(t)) x(t)+B u(t)+f(t, x), \\
y(t)=C x(t)+C_{d} d(t),
\end{gathered}
$$

where $A \in R^{n \times n}, B \in R^{n \times m}, C \in R^{P \times n}$, and $C_{d} \in R^{P \times d}$.

Throughout this paper, we first provide the following assumptions for the following proof

(i) $(A, B)$ is a stabilizable pair, $(A, C)$ is an observable pair, and $C_{d}$ is a full column rank;

(ii) $f(t, x)$ satisfies the following Lipschitz condition:

$\|f(t, x)-f(t, \widehat{x})\| \leq \theta\|x-\widehat{x}\|, \quad$ for $\forall x(t), \widehat{x}(t) \in R^{n}$;

(iii) $d(t)$ satisfies the norm-bounded condition $\|d(t)\| \leq \delta$, where $\delta>0$ is known constant;

(iv) $\triangle A(t)$ is unknown time-varying uncertainties satisfying $\Delta A(t)=D_{1} \cdot F(t) \cdot D_{2}$, with $D_{1} \in R^{n \times n}, D_{2} \in R^{n \times n}$, and $F(t) \in R^{n \times n}$ is the unknown time-varying and satisfies $F^{T}(t) \cdot F(t) \leq I_{n}$.

\section{Main Results}

Consider the following traditional Luenberger observer:

$$
\dot{\hat{x}}(t)=(A-L) \cdot \hat{x}(t)+B u(t)+L y(t)+f(t, \widehat{x}),
$$

where $\hat{x}(t)$ is the estimation of $x(t)$ and $L$ is the observer gain to be designed. If one employs the observer (10) to plant (8) and defines $e(t)=\hat{x}(t)-x(t)$, then one can obtain the following error dynamics:

$$
\begin{aligned}
\dot{e}(t)= & {[A-L+\Delta A(t)] \cdot e(t)-\Delta A(t) \cdot \hat{x}(t) } \\
& +L C_{d} d(t)+f(t, e(t)),
\end{aligned}
$$

where $f(t, e(t))=f(t, \widehat{x}(t))-f(t, x(t))$. It is observed that the disturbance $C_{d} d(t)$ in the error system (11) is multiplied 
by the gain matrix $L$. Hence, if $L$ is a high gain, the disturbance effect will be amplified unavoidably. As a result, ideal estimation performance of system state $x(t)$ cannot be obtained by using the conventional observer (10).

To begin the formulation of our approach, the following augmented variables and matrices are defined:

$$
\begin{aligned}
& \bar{x}(t)=\left[\begin{array}{c}
x(t) \\
C_{d} d(t)
\end{array}\right], \quad \bar{A}=\left[\begin{array}{cc}
A & 0 \\
0 & -I_{P}
\end{array}\right], \\
& \bar{B}=\left[\begin{array}{c}
B \\
0_{p \times m}
\end{array}\right], \quad \bar{C}=\left[\begin{array}{ll}
C & I_{P \times P}
\end{array}\right], \\
& \bar{E}=\left[\begin{array}{cc}
I_{n} & 0 \\
0 & 0_{P \times P}
\end{array}\right], \quad \bar{F}=\left[\begin{array}{cc}
F(t) & 0_{n \times P} \\
0_{P \times n} & 0_{P \times P}
\end{array}\right] \text {, } \\
& \bar{D}_{1}=\left[\begin{array}{cc}
D_{1} & 0 \\
0 & 0_{P \times P}
\end{array}\right], \quad \bar{D}_{2}=\left[\begin{array}{cc}
D_{2} & 0 \\
0 & 0_{P \times P}
\end{array}\right] \text {, } \\
& \Delta \bar{A}(t)=\left[\begin{array}{cc}
\Delta A(t) & 0 \\
0 & 0_{P \times P}
\end{array}\right], \quad \bar{N}=\left[\begin{array}{c}
0 \\
I_{P}
\end{array}\right] \text {, } \\
& \bar{f}(t, x)=\left[\begin{array}{c}
f(t, x) \\
0_{P \times 1}
\end{array}\right],
\end{aligned}
$$

and the following augmented plant is constructed:

$$
\begin{gathered}
\bar{E} \dot{\bar{x}}(t)=[\bar{A}+\Delta \bar{A}(t)] \bar{x}(t)+\bar{B} u(t)+\bar{f}(t, e)+\bar{N} d(t), \\
y(t)=\bar{C} \bar{x}(t) .
\end{gathered}
$$

System (13) is a singular system, where the state vector $x(t)$ and disturbance vector $d(t)$ are both the components of the augmented state vector.

It is noted that $\bar{E}$ and $\bar{C}$ defined previously have the following property:

$$
\operatorname{rank}\left[\frac{\bar{E}}{\bar{C}}\right]=\operatorname{rank}\left[\begin{array}{cc}
I_{n} & 0 \\
0 & 0_{P \times P} \\
C & I_{P}
\end{array}\right]=n+P .
$$

Therefore, an appropriate matrix $L_{D} \in R^{(n+p) \times p}$ can always be selected such that the matrix $\bar{S} \triangleq\left(\bar{E}+\bar{L}_{D} \bar{C}\right)$ is nonsingular. Motivated by this observation, the following proportional and derivative observer is proposed for system (13):

$$
\begin{gathered}
\bar{S} \dot{\bar{z}}(t)=\left(\bar{A}-\bar{L}_{P} \bar{C}\right) \bar{z}(t)+\overline{A S}^{-1} \bar{L}_{D} y(t)+\bar{B} u(t)+\bar{f}(t, \widehat{x}), \\
\widehat{\bar{x}}(t)=\bar{z}(t)+\bar{S}^{-1} \bar{L}_{D} y(t)
\end{gathered}
$$

where $\bar{z}(t)=\left[\bar{z}_{x}^{T}(t) \bar{z}_{d}^{T}(t)\right]^{T}$ is the middle variable, $\widehat{\bar{x}}(t) \triangleq$ $\left[\widehat{\bar{x}}^{T}(t) \widehat{d}^{T}(t)\right]^{T}$ is the estimation of $\bar{x}(t)$, and $\bar{L}_{D} \in R^{(n+p) \times p}$ and $\bar{L}_{p} \in R^{(n+p) \times p}$ are the observer gains to be designed. $\bar{S}=$ $\bar{E}+\bar{L}_{D} \cdot \bar{C}$ is designed to be invertible by selecting appropriate
$\bar{L}_{D}$. It is now in the position to derive the error system. In fact, from (14)-(15), it is derived that

$$
\begin{aligned}
\bar{S} \dot{\bar{x}}= & \left(\bar{A}-\bar{L}_{P} \bar{C}\right) \hat{\bar{x}}(t)+\bar{L}_{P} y(t) \\
& +\bar{L}_{D} \dot{y}(t)+\bar{B} u(t)+\bar{f}(t, \widehat{x}) .
\end{aligned}
$$

On the other hand, adding $\bar{L}_{D} \dot{y}(t)$ to both sides of the plant (13), the following equation is derived:

$$
\begin{aligned}
\bar{S} \dot{\bar{x}}(t)= & {\left[\left(\bar{A}-\bar{L}_{P} \bar{C}\right)+\Delta A(t)\right] \bar{x}(t)+\bar{L}_{P} \dot{y}(t) } \\
& +\bar{L}_{D} \dot{\bar{y}}(t)+\bar{B} u(t)+\bar{f}(t, x)+\bar{N} d(t) .
\end{aligned}
$$

Define the following error variable:

$$
\bar{e}(t)=\widehat{\bar{x}}(t)-\bar{x}(t), \quad \bar{f}(t, \bar{e})=\bar{f}(t, \widehat{x})-\bar{f}(t, x) .
$$

Subtracting (17) from (16), the error system is derived as

$$
\bar{S} \dot{\bar{e}}(t)=\left[\bar{A}-\bar{L}_{P} \bar{C}\right] \bar{e}(t)-\Delta \bar{A}(t) \bar{x}(t)-\bar{N} d(t)+\bar{f}(t, \bar{e}) .
$$

On the other hand, we propose the following observerbased control strategy for plant (8):

$$
u(t)=\bar{K} \hat{\bar{x}}(t) \quad K \in R^{m \times(n+p)},
$$

where $\bar{K}=\left[K, 0_{m \times p}\right]$. Applying (20) to plant (8) yields

$$
\begin{aligned}
\dot{x}(t) & =(A+\Delta A(t)) x(t)+B \bar{K} \hat{\bar{x}}(t)+f(t) \\
& =(A+\Delta A(t)) x(t)+B \bar{K}(\bar{e}(t)+\bar{x}(t))+f(t) \\
& =(A+\Delta A(t)) x(t)+B \bar{K} \bar{e}(t)+B \bar{K} \bar{x}(t)+f(t) .
\end{aligned}
$$

Equation (21) together with (19) yields the following overall closed-loop system:

$$
\begin{gathered}
\dot{x}(t)=(A+\Delta A(t)) x(t)+B K \bar{x}(t)+f(t)+B \bar{K} \bar{e}(t), \\
\dot{e}(t)=\bar{S}^{-1}\left[\bar{A}-\bar{L}_{P} \bar{C}\right] \bar{e}(t)-\Delta \bar{A}(t) \bar{x}(t)+\bar{N} d(t)+\bar{f}(t, \bar{e}) .
\end{gathered}
$$

In system (22), recall that $\bar{S}=\left(\bar{E}+\bar{L}_{D} \bar{C}\right)$. We decompose $\bar{L}_{D}$ as $\bar{L}_{D}=\left[\begin{array}{l}\bar{L}_{D_{1}} \\ \bar{L}_{D_{2}}\end{array}\right]$ with $\bar{L}_{D_{1}} \in R^{n \times p}, \bar{L}_{D_{2}} \in R^{P \times P}$. In this paper, let $\bar{L}_{D_{1}}=0, \bar{L}_{D_{2}}=\operatorname{diag}\left\{\beta_{1}, \ldots, \beta_{P}\right\}$, where $\beta_{1} \cdots \beta_{P}$ are reasonable large numbers. In this setting, the derivative gain $\bar{L}_{D}=\left[\begin{array}{c}0 \\ L_{D_{2}}\end{array}\right]$ can thus guarantee that $\bar{S}=\bar{E}+\bar{L}_{D} \bar{C}$ is nonsingular and reduce the effect of disturbance $f(t)$ at the same time. In addition, design the state feedback gain $K \in$ $R^{m \times n}$, such that $A+B K$ is Hurwitz. The following theorem provides a sufficient condition for the stability analysis of the overall closed-loop system (22).

Theorem 1. Consider the overall closed-loop system (22), where the observer gain $\bar{L}_{D}$ and feedback gain $K$ have been designed as previously discussed. If there exist positive and 
definite matrices $P \in R^{n \times n}, \bar{R} \in R^{(n+p) \times(n+p)}$, and matrices $\bar{L}_{p} \in R^{(n+p) \times p}$, such that the following matrix condition:

$$
\left[\begin{array}{cccccc}
\Phi_{11} & P B K & 0 & P D_{1} & \theta P & 0 \\
* & \Phi_{22} & \overline{R S}^{-1} \bar{D}_{1} & 0 & 0 & \overline{R S}^{-1} \\
* & * & -\varepsilon_{1} I_{n} & 0 & 0 & 0 \\
* & * & * & -\varepsilon_{2} I_{n+p} & 0 & 0 \\
* & * & * & * & -I_{n} & 0 \\
* & * & * & * & * & -I_{n+p}
\end{array}\right]<0
$$

with $\Phi_{11}=(A+B K)^{T} P+P(A+B K)+I_{n}+\theta I_{n}+\varepsilon_{2} D_{2}^{T} D_{2}$, $\Phi_{22}=\overline{R S}^{-1}\left(\bar{A}-\bar{L}_{P} \bar{C}\right)+\left(\bar{A}-\bar{L}_{P} \bar{C}\right) \bar{S}^{-T} \bar{R}$.

Proof. We define the following Lyapunov function: $V(t)=$ $V_{1}(t)+V_{2}(t)$ with $V_{1}(t)=x^{T}(t) P x(t), V_{2}(t)=\bar{e}^{T}(t) \bar{R} \bar{e}(t)$ for system (22). Along the trajectory of system (22), it follows that

$$
\begin{array}{r}
\dot{V}_{1}(t)=2 x^{T}(t) P[(A+\Delta A(t)) x(t)+B K x(t)+f(t)] \\
\dot{V}_{2}(t)=2 \bar{e}^{T}(t) \bar{R}\left[\bar{S}^{-1}\left(\bar{A}-\bar{L}_{P} \bar{C}\right)-\Delta \bar{A}(t) \bar{x}(t)\right. \\
+\bar{N} d(t)+\bar{f}(t, \bar{e})] \\
=2 \bar{e}^{-T}(t) \bar{R}\left[\bar{S}^{-1}\left(\bar{A}-\bar{L}_{P} \bar{C}\right) \bar{e}(t)-\Delta \bar{A}(t) \bar{x}(t)\right] \\
\quad-2 \bar{e}^{T}(t) \overline{R S}^{-1} \bar{N} d(t)+2 \bar{e}^{T}(t) \overline{R S}^{-1} \bar{f}(t, \bar{e}) .
\end{array}
$$

Since $\bar{S}=\left[\begin{array}{cc}I_{n}+\bar{L}_{D 1} C & \bar{L}_{D 1} \\ \bar{L}_{D 2} C & \bar{L}_{D 2}\end{array}\right]$, one can calculate that $\bar{S}^{-1}=$ $\left[\begin{array}{lc}I_{n} & 0 \\ -C & \left(\bar{L}_{D 2}\right)^{-1}\end{array}\right]$.

Hence, it is calculated that $\bar{S}^{-1} \bar{N} d(t)=$ $\left[\begin{array}{cc}I_{n} & 0 \\ -C & \left(\bar{L}_{D 2}\right)^{-1}\end{array}\right]\left[\begin{array}{c}0 \\ I_{P}\end{array}\right] d(t)=\left[\begin{array}{c}0 \\ \left(\bar{L}_{D 2}\right)^{-1}\end{array}\right] d(t)$. Since $\left(\bar{L}_{D 2}\right)^{-1}=$ $\operatorname{diag}\left\{\beta_{1}^{-1}, \ldots, \beta_{p}^{-1}\right\}$, the term $\bar{S}^{-1} \bar{N} d(t)$ is prevailed successfully and can be ignored in $\dot{V}_{2}(t)$. Therefore, $\dot{V}_{2}(t)$ becomes

$$
\begin{aligned}
\dot{V}_{2}(t)= & 2 \bar{e}^{T}(t) \bar{R}\left[\bar{S}^{-1}\left(\bar{A}-\bar{L}_{P} \bar{C}\right) \bar{e}^{T}(t)-\Delta \bar{A}(t) \bar{x}(t)\right] \\
& +2 \bar{e}^{T}(t) \overline{R S}^{-1} \bar{f}(t, \bar{e}) .
\end{aligned}
$$

Notice that

$$
\begin{aligned}
2 \bar{e}^{T}(t) \overline{R S}^{-1} \bar{f}(t, \bar{e}) & \leq 2 \bar{e}^{T}(t) \overline{R S}^{-1} \bar{S}^{-T} \bar{R} \bar{e}(t)+\bar{f}^{T}(t, \bar{e}) \bar{f}(t, \bar{e}) \\
& \leq 2 \bar{e}^{T}(t) \overline{R S}^{-1} \bar{S}^{-T} \bar{R}^{-T}(t)+\bar{f}^{T}(t, e) f(t, e) \\
& \leq 2 \bar{e}^{T}(t) \overline{R S}^{-1} \bar{S}^{-T} \bar{R} \bar{e}(t)+\theta^{2} \cdot x^{T}(t) x(t) .
\end{aligned}
$$

It is thus derived from (25) and (26) that

$$
\begin{aligned}
\dot{V}_{2}(t) \leq & 2 \bar{e}^{T}(t) \bar{R}\left[\bar{S}^{-1}\left(\bar{A}-\bar{L}_{P} \bar{C}\right) \bar{e}(t)-\bar{S}^{-1} \Delta \bar{A}(t) \bar{x}(t)\right] \\
& +2 \bar{e}^{T}(t) \overline{R S}^{-1} \bar{S}^{-T} \bar{R} \bar{e}(t)+\theta^{2} \cdot x^{T}(t) x(t) .
\end{aligned}
$$

Notice that

$$
\begin{aligned}
\Delta \bar{A}(t) \bar{x}(t) & =\left[\begin{array}{cc}
\Delta A(t) & 0 \\
0 & 0
\end{array}\right]\left[\begin{array}{c}
x(t) \\
C_{s} d(t)
\end{array}\right] \\
& =\left[\begin{array}{cc}
D_{1} F(t) D_{2} & 0_{n \times p} \\
0_{p \times n} & 0_{p \times p}
\end{array}\right]\left[\begin{array}{c}
x(t) \\
C_{s} d(t)
\end{array}\right],
\end{aligned}
$$

and also recall that $\bar{D}_{1}=\left[\begin{array}{cc}D_{1} & 0_{n \times p} \\ 0_{p \times n} & 0_{p \times p}\end{array}\right], \bar{D}_{2}=\left[\begin{array}{cc}D_{2} & 0_{n \times p} \\ 0_{p \times n} & 0_{p \times p}\end{array}\right]$, then it is shown that

$$
\begin{aligned}
\Delta \bar{A}(t) \bar{x}(t)= & {\left[\begin{array}{cc}
D_{1} & 0_{n \times p} \\
0_{p \times n} & 0_{p \times p}
\end{array}\right]\left[\begin{array}{cc}
F(t) & 0_{n \times p} \\
0_{p \times n} & 0_{p \times p}
\end{array}\right] } \\
& \times\left[\begin{array}{cc}
D_{2} & 0_{n \times p} \\
0_{p \times n} & 0_{p \times p}
\end{array}\right]=\bar{D}_{1} \bar{F}(t) \bar{D}_{2} .
\end{aligned}
$$

And there exists $\varepsilon_{1}>0$, such that

$$
\begin{aligned}
-2 \bar{e}^{T}(t) \overline{R S}^{-1} \Delta \bar{A}(t) \bar{x}(t)= & -2 \bar{e}^{-T}(t) \overline{R S}^{-1} \bar{D}_{1} \bar{F}(t) \bar{D}_{2} \bar{x}(t) \\
\leq & \varepsilon_{1} \bar{x}^{T}(t) \bar{D}_{2}^{T} \bar{D}_{2} \bar{x}(t) \\
& +\varepsilon_{1}^{-1} \bar{e}^{T}(t) \overline{R S}^{-1} \bar{D}_{1} \bar{D}_{1}^{T} \bar{S}^{-T} \bar{R} \bar{e}(t) \\
\leq & \varepsilon_{1} x^{T}(t) D_{2}^{T} D_{2} x(t) \\
& +\varepsilon_{1}{ }^{-1} \bar{e}^{T}(t) \overline{R S}^{-1} \bar{D}_{1} \bar{D}_{1}^{T} \bar{S}^{-T} \bar{R} \bar{e}(t) .
\end{aligned}
$$

As a result, it is derived from (27) and (30) that

$$
\begin{aligned}
\dot{V}_{2}(t) \leq & 2 \bar{e}^{T}(t) \overline{R S}^{-1}\left(\bar{A}-\bar{L}_{P} \bar{C}\right)+\varepsilon_{1} x^{T}(t) D_{2}^{T} D_{2} x(t) \\
& +\varepsilon_{1}^{-1} \bar{e}^{T}(t) \overline{R S}^{-1} \bar{D}_{1} \bar{D}_{1}^{T} \bar{S}^{-T} \bar{R} \bar{e}(t) \\
& +2 \bar{e}^{T}(t) \overline{R S}^{-1} \bar{S}^{-T} \bar{R} \bar{e}(t)+\theta^{2} \cdot x^{T}(t) x(t) .
\end{aligned}
$$

On the other hand, for $\dot{V}_{1}(t)$, we have

$$
\begin{aligned}
2 x^{T}(t) P \Delta A(t) x(t)= & 2 x^{T}(t) P D_{1} F(t) D_{2} x(t) \\
\leq & \varepsilon_{2} x^{T}(t) D_{2}^{T} D_{2} x(t) \\
& +\varepsilon_{2}{ }^{-1} x^{T}(t) P D_{1} D_{1}^{T} P x(t),
\end{aligned}
$$

and $2 x^{T}(t) P f(t) \leq 2\|x(t)\|^{2}\|P\| \cdot \theta \leq \theta^{2} \cdot x^{T}(t) P P x(t)+$ $x^{T}(t) x(t)$.

Then

$$
\begin{aligned}
\dot{V}_{1}(t) \leq & 2 x^{T}(t) P(A+B K) x(t)+\varepsilon_{2} x^{T}(t) D_{2}^{T} D_{2} x(t) \\
& +\varepsilon_{2}^{-1} x(t) P D_{1} D_{1}^{T} P x_{1}(t) \\
& +\theta^{2} \cdot x^{T}(t) P P x(t)+x^{T}(t) x(t) \\
& +2 x^{T}(t) P B K x(t) .
\end{aligned}
$$


As a result, it is derived that

$$
\begin{aligned}
\dot{V}(t)= & \dot{V}_{1}(t)+\dot{V}_{2}(t) \\
\leq & 2 x^{T}(t) P(A+B K) x(t)+\varepsilon_{2} x^{T}(t) D_{2}^{T} D_{2} x(t) \\
& +\varepsilon_{2}^{-1} x^{T}(t) P D_{1} D_{1}^{T} P x_{1}(t)+\theta^{2} \cdot x^{T}(t) P P x(t) \\
& +x^{T}(t) x(t)+2 x^{T}(t) P B K x(t) \\
& +2 \bar{e}^{T}(t) \overline{R S}^{-1}\left(\bar{A}-\bar{L}_{P} \bar{C}\right)+\varepsilon_{1} x^{T}(t) \\
& \times D_{2}^{T} D_{2} x(t)+\varepsilon_{1}^{-1} \bar{e}^{T}(t) \overline{R S}^{-1} \bar{D}_{1} \bar{D}_{1}^{T} \bar{S}^{-T} \bar{R} \bar{e}(t) \\
& +2 \bar{e}^{T}(t) \overline{R S}^{-1} \bar{S}^{-T} \bar{R}_{\bar{e}}(t)+\theta^{2} \cdot x^{T}(t) x(t) \\
\leq & {\left[x^{T}(t) \bar{e}^{T}(t)\right]\left[\begin{array}{cc}
\mathrm{II}_{11} & \mathrm{II}_{12} \\
* & \mathrm{II}_{22}
\end{array}\right]\left[\begin{array}{l}
x \\
\bar{e}(t)
\end{array}\right] }
\end{aligned}
$$

with

$$
\begin{aligned}
\mathrm{II}_{11}= & (A+B K)^{T} P+P(A+B K)+\varepsilon_{2} D_{2}^{T} D_{2} \\
& +\varepsilon_{2}^{-1} P D_{1} D_{1}^{T} P+\theta^{2} P P+I_{n}+\theta^{2} I_{n}, \\
\mathrm{II}_{22}= & \overline{R S}^{-1}\left(\bar{A}-\bar{L}_{P} \bar{C}\right)+\left(\bar{A}-\bar{L}_{P} \bar{C}\right)^{T} \bar{S}^{-T} \bar{R} \\
& +\varepsilon_{1}^{-1} \overline{R S}^{-1} \bar{D}_{1} \bar{D}_{1}^{T} \bar{S}^{-T} \bar{R}+\overline{R S}^{-1} \bar{S}^{-T} \bar{R} .
\end{aligned}
$$

By Schur complement lemma, II $<0$ is equivalent to the matrix condition (23) in Theorem 1 . This completes the proof.

It is noted that the matrix condition (23) in Theorem 1 is a nonlinear one since the observer gain $\bar{L}_{P}$ has not been designed. To this end, we provide the following theorem to design $\bar{L}_{P}$ and transform the condition of Theorem 1 into a linear version.

Theorem 2. For the closed-loop system (22), let the gain matrices $\bar{L}_{D}$ and $K$ be designed as previously discussed, if there exist positive and definite matrix $P \in R^{n \times n}, \bar{R} \in R^{(n+p) \times(n+p)}$, and matrices $\bar{Y} \in R^{(n+p) \times p}$ such that the following LMI holds:

$$
\left[\begin{array}{cccccc}
\Psi_{11} & P B K & 0 & P D_{1} & \theta P & 0 \\
* & \Psi_{22} & \overline{R S}^{-1} \bar{D}_{1} & 0 & 0 & \overline{R S}^{-1} \\
* & * & -\varepsilon_{1} I_{n} & 0 & 0 & 0 \\
* & * & * & -\varepsilon_{2} I_{n+p} & 0 & 0 \\
* & * & * & * & -I_{n} & 0 \\
* & * & * & * & * & -I_{n+p}
\end{array}\right]<0
$$

where

$$
\begin{gathered}
\Psi_{11}=(A+B K)^{T} P+P(A+B K)+I_{n}+\theta I_{n}+\varepsilon_{2} D_{2}^{T} D_{2} \\
\Psi_{22}=\overline{R S A}-\bar{Y} C+\bar{A}^{T} \bar{S}^{T} \bar{R}-C^{T} \bar{Y}^{T} .
\end{gathered}
$$

Furthermore, the observer gain $\bar{L}_{P}$ is calculated as $\bar{L}_{P}=$ $\overline{S R}^{-1} \bar{Y}$.
TABLE 1: Miscellaneous coefficient value.

\begin{tabular}{lcc}
\hline Coefficients & Value & Unit \\
\hline$K_{i}$ & $1 e-3$ & $\mathrm{~A} / \mathrm{V}$ \\
$K_{s}$ & 1.5 & $\mathrm{~m} / \mathrm{A}$ \\
$A_{g}$ & $1.8 e-3$ & $\mathrm{~m}^{2}$ \\
$\beta_{e}$ & $1 e 9$ & $\mathrm{~Pa}$ \\
$C_{i c}$ & $2.4 e-11$ & $\mathrm{~m} \cdot \mathrm{N} / \mathrm{s}$ \\
$C_{e c}$ & $7.3 e-13$ & $\mathrm{~m} \cdot \mathrm{N} / \mathrm{s}$ \\
$V_{t}$ & $1 e-4$ & $\mathrm{~m}^{3}$ \\
$m$ & 3.75 & $\mathrm{~kg}$ \\
$B_{c}$ & 500 & $\mathrm{~N} \cdot \mathrm{s} / \mathrm{m}$ \\
$K$ & $1.66 e 5$ & $\mathrm{~N} / \mathrm{m}$ \\
$D_{\max }$ & $2.39 e-5$ & $\mathrm{~m} / \mathrm{rad}$ \\
$y_{\max }$ & 0.015 & $\mathrm{~m}$ \\
$C_{t c}$ & $2.4 e-11$ & $\mathrm{~m} \cdot \mathrm{N} / \mathrm{s}$ \\
$B$ & 0.09 & $\mathrm{~N} \cdot \mathrm{m} /(\mathrm{rad} / \mathrm{s})$ \\
$J_{p / m}$ & 0.52 & $\mathrm{~kg} \cdot \mathrm{m}^{2}$ \\
$K_{q}$ & $5.56 e-3$ & $(\mathrm{~m} / \mathrm{s}) / \mathrm{m}$ \\
$K_{c}$ & $8 e-12$ & $\mathrm{~m} / \mathrm{N} \cdot \mathrm{s}$ \\
$r$ & 145.2 & - \\
$P_{s}$ & $15 e 6$ & $\mathrm{~Pa}$ \\
\hline
\end{tabular}

Proof. In the matrix condition of Theorem 1, let $\bar{Y}=\overline{R S}^{-1} \bar{L}_{p}$, and substituting it into the matrix condition of Theorem 1 , one can easily obtain the LMI of Theorem 2 . This completes the proof.

\section{Simulation Results}

Consider the plant and augmented form (8), the system data are chosen as shown in Table 1 as follows:

$$
\begin{aligned}
& A=\left(\begin{array}{cccc}
-0.173 & 4.59 \times 10^{4} & 0 & 0 \\
0 & 0 & 1 & 0 \\
0 & -4.43 \times 10^{4} & -133.3 & 4.8 \times 10^{-4} \\
0 & 0 & -7.14 \times 10^{10} & -1.28 \times 10^{3}
\end{array}\right) \text {, } \\
& B=\left(\begin{array}{c}
0 \\
0 \\
0 \\
3.3 \times 10^{8}
\end{array}\right), \quad C=\left(\begin{array}{l}
1 \\
0 \\
0 \\
0
\end{array}\right)^{T} \\
& D_{1}=\left[\begin{array}{cccc}
0.1 & 0.2 & 0.05 & 0.1 \\
0 & 0.2 & 0.1 & 0.1 \\
0 & 0.1 & 0.13 & 0.1 \\
0.13 & 0.12 & 0.14 & 0.15
\end{array}\right] \\
& D_{2}=\left[\begin{array}{cccc}
0.2 & 0.1 & 0.07 & 0.02 \\
0 & 0.1 & 0.2 & 0.3 \\
0.1 & 0.1 & 0.13 & 0.1 \\
0.14 & 0.12 & 0.14 & 0.15
\end{array}\right] \\
& F(t)=\left[\begin{array}{cccc}
0.1 \sin (t) & 0 & 0 & 0 \\
0 & 0.15 \sin (t) & 0 & 0 \\
0 & 0 & 0.14 \sin (t) & 0 \\
0 & 0 & 0 & 0.1 \sin (t)
\end{array}\right] \text {, }
\end{aligned}
$$


and the Lipschitz condition is selected as

$$
f(t, x)=0.131\left[\begin{array}{c}
\sin \left(-0.2 x_{1}(t)+x_{2}(t)+x_{3}(t)\right) \\
\sin \left(x_{1}(t)-2 x_{2}(t)+x_{3}(t)\right) \\
0 \\
0
\end{array}\right]
$$

It is easy to check that $(A, C)$ is observable pair, and the Lipschitz condition is given as $\theta=0.420$. It is assumed that $d(t)$ has the following form.

Case 1. Consider

$$
d(t)= \begin{cases}0.1 \cos (8 t), & t<2, \\ 0.1 \sin (10 t)+0.2 \cos (10 t), & 2 \leq t<5\end{cases}
$$

Case 2. Consider

$$
d(t)= \begin{cases}0.5, & t<3 \\ 0.3 \sin (4 t)+0.1, & 3 \leq t<5 .\end{cases}
$$

We select $\delta=0.4$, which is the norm upper bound of $d(t)$ as defined in assumption (iii). In order to design the estimator, we choose the derivative gain $\bar{L}_{D}$ as

$$
\begin{aligned}
\bar{L}_{D} & =\left[\begin{array}{l}
0 \\
0 \\
0 \\
1
\end{array}\right] \text {, such that the system matrix } \\
\bar{S} & =\left[\begin{array}{lllll}
1 & 0 & 0 & 0 & 0 \\
0 & 1 & 0 & 0 & 0 \\
0 & 0 & 1 & 0 & 0 \\
0 & 0 & 0 & 1 & 0 \\
1 & 0 & 0 & 0 & 1
\end{array}\right] \text { is nonsingular. }
\end{aligned}
$$

Design the state feedback gain as $K=[-1.413,-0.286$, $-2.854,-0.423$ ] such that $A+B K$ is Hurwitz. Solving the LMI condition of Theorem 2, one can obtain the following solution:

$$
\begin{aligned}
P & =\left[\begin{array}{ccccc}
369.0123 & -254.7902 & -107.2517 & -184.3887 \\
254.7902 & 352.6723 & -104.8523 & 75.6133 \\
-107.2517 & -104.8523 & 502.8899 & 134.2559 \\
-184.3887 & 75.6133 & 134.2559 & 212.2635
\end{array}\right], \\
R & =\left[\begin{array}{ccccc}
3.6029 & -3.7781 & 5.8391 & -1.3649 & 1.2867 \\
-3.7781 & 9.2899 & -13.8548 & 0.7897 & -3.7774 \\
5.8391 & -13.8548 & 29.0279 & -0.2689 & 6.1213 \\
-1.3649 & 0.7897 & -0.2689 & 1.0889 & 0.3238 \\
1.2867 & -3.7774 & 6.1213 & 0.3238 & 2.2892
\end{array}\right] .
\end{aligned}
$$

According to Theorem 2, the proportional gain is selected as

$$
\bar{L}_{p}=10^{2} \times\left[\begin{array}{l}
0.0000 \\
0.0000 \\
0.0000 \\
6.3696 \\
0.0000
\end{array}\right] \text {. }
$$

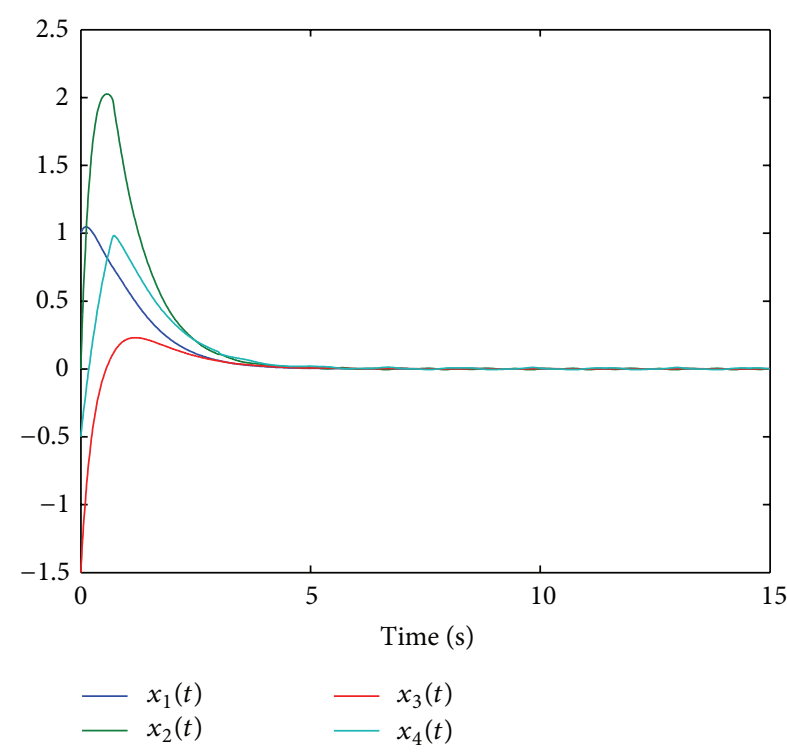

FIGURE 2: Trajectory of the closed-loop system.

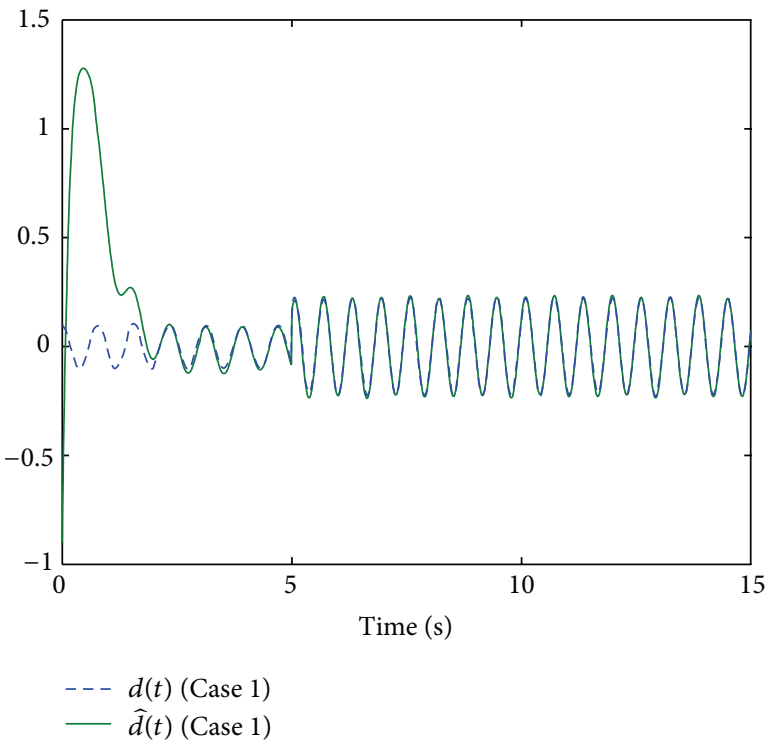

FIgURE 3: $d(t)$ and the estimation: Case 1.

The initial condition is chosen as $\bar{e}(1)=$ $\left[\begin{array}{lllll}0 & 0 & 0 & 0 & 0\end{array}\right], x(1)=\left[\begin{array}{llll}1 & -1 & -1.5 & 1\end{array}\right]$. The simulation results are provided in Figures 1-3. The state trajectory of $x(t)$ of the closed-loop system is shown in Figure 2. The trajectories of disturbances $d(t)$ and their estimation in Case 1 are shown in Figure 3. The trajectories of disturbances $d(t)$ and their estimation in Case 2 are shown in Figure 4. It can be seen that the asymptotic stability of the closed-loop system is guaranteed, and the tracking performance of system states and disturbances has achieved an ideal performance.

\section{Conclusion}

In this paper, the problem of robust control has been studied for the velocity control system based on CPR with parameters uncertainty and load disturbance. The hydraulic 


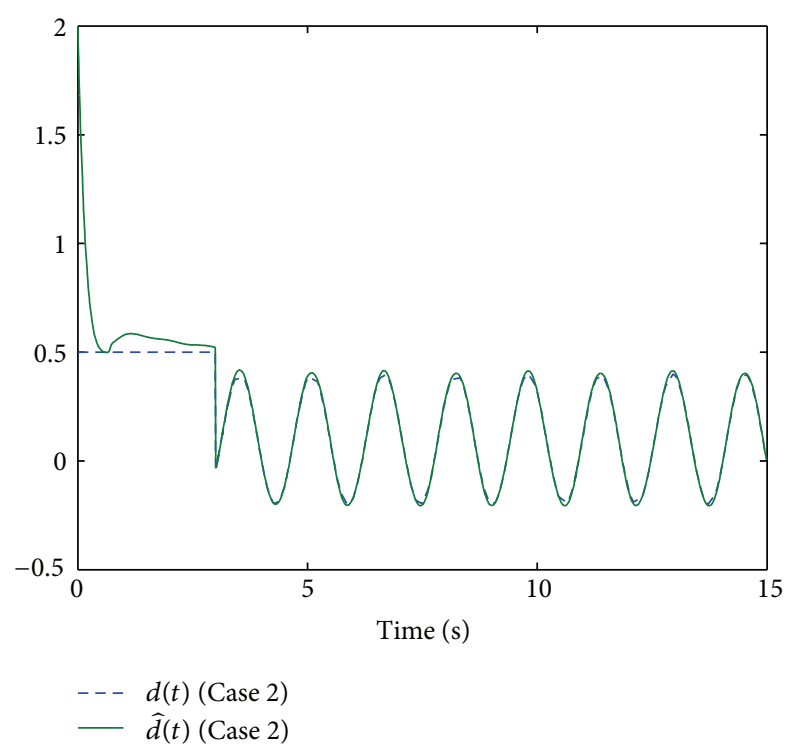

FIgure 4: $d(t)$ and the estimation: Case 2.

velocity control system model has been built firstly for the control design objective. By constructing a Luenberger observer and using Lyapunov stability theory, the observerbased controller has been designed such that the closed-loop system is asymptotically stable and satisfies the disturbance attenuation level. Finally, simulation results have shown the effectiveness of the proposed method. The future work will focus on constructing one test rig and trying to prove the effective performance for the proposed control method. And the long research object of the project is to apply this method to hydraulic velocity control system design for getting a good performance of construction machinery.

\section{Acknowledgments}

The authors acknowledge the contribution of the National Natural Science Foundation of China (50875054) and Foreign Visiting Project of Harbin Institute of Technology for Ph.D. Students.

\section{References}

[1] D. Wang, C. Guan, S. Pan, M. Zhang, and X. Lin, "Performance analysis of hydraulic excavator powertrain hybridization," Automation in Construction, vol. 18, no. 3, pp. 249-257, 2009.

[2] P. A. J. Achten and J. O. Palmberg, "What a difference a hole makes: the commercial value of the innas hydraulic transformer," in Proceedings of the 6th Scandinavian International Conference on Fluid Power, pp. 873-886, 1999.

[3] P. Achten, G. Vael, H. Murrenhoff, T. Kohmäscher, and M. Inderelst, "Low-emission hydraulic hybrid for passenger cars," Automobiltechnische Zeitschrift, vol. 111, pp. 378-384, 2009.

[4] C. N. Okoye, J. Jiang, and L. Y. Hui, “Design and development of secondary controlled industrial palm kernel nut vegetable oil expeller plant for energy saving and recuperation," Journal of Food Engineering, vol. 87, no. 4, pp. 578-590, 2008.
[5] W. Shen and J. Jiang, "Study on control performance and energy recovery efficiency of hydraulic hybrid excavator," ICIC Express Letters, vol. 5, pp. 4439-4444, 2011.

[6] C. S. Kim and C. O. Lee, "Robust speed control of a variabledisplacement hydraulic motor considering saturation nonlinearity," Journal of Dynamic Systems, Measurement and Control, vol. 122, no. 1, pp. 196-201, 2000.

[7] T. Radpukdee and P. Jirawattana, "Uncertainty learning and compensation: an application to pressure tracking of an electrohydraulic proportional relief valve," Control Engineering Practice, vol. 17, no. 2, pp. 291-301, 2009.

[8] J. Jiang, Y. Han, and D. Wang, "The intelligent PID control in Hydrostatic drive system with secondary regulation," Journal of Harbin Institute of Technology, vol. 30, pp. 36-38, 1998.

[9] Q. Zhou, P. Shi, J. Lu, and S. Xu, "Adaptive output feedback fuzzy tracking control for a class of nonlinear systems," IEEE Transactions on Fuzzy Systems, vol. 19, no. 5, pp. 972-982, 2011.

[10] Y. Tang, H. Gao, W. Zou, and J. Kurths, "Distributed synchronization in networks of agent systems with nonlinearities and random switchings," IEEE Transactions on Systems, Man, and Cybernetics B, vol. 43, no. 1, pp. 358-370, 2013.

[11] H. Li, H. Liu, H. Gao, and P. Shi, "Reliable fuzzy control for active suspension systems with actuator delay and fault," IEEE Transactions on Fuzzy Systems, vol. 20, no. 2, pp. 342-357, 2012.

[12] H. Gao and T. Chen, " $H_{\infty}$ estimation for uncertain systems with limited communication capacity," IEEE Transactions on Automatic Control, vol. 52, no. 11, pp. 2070-2084, 2007.

[13] H. Gao, J. Lam, and C. Wang, "Multi-objective control of vehicle active suspension systems via load-dependent controllers," Journal of Sound and Vibration, vol. 290, no. 3-5, pp. 654-675, 2006.

[14] S. Zhou and W. X. Zheng, "Robust $H_{\infty}$ control of delayed singular systems with linear fractional parametric uncertainties," Journal of the Franklin Institute, vol. 346, no. 2, pp. 147-158, 2009.

[15] X. Yang, H. Gao, and P. Shi, "Robust orbital transfer for low earth orbit spacecraft with small-thrust," Journal of the Franklin Institute, vol. 347, no. 10, pp. 1863-1887, 2010.

[16] M. V. Basin, P. Soto, and D. Calderon-Alvarez, "Central suboptimal $H_{\infty}$ controller design for linear time-varying systems with unknown parameters," International Journal of Systems Science, vol. 42, no. 5, pp. 709-716, 2011.

[17] H. Gao, J. Lam, and Z. Wang, "Discrete bilinear stochastic systems with time-varying delay: stability analysis and control synthesis," Chaos, Solitons and Fractals, vol. 34, no. 2, pp. 394404, 2007.

[18] E. Tian, D. Yue, and C. Peng, "Delay-fractioning-dependent robust $H_{\infty}$ control for linear systems with delay in state or input and its applications," International Journal of Systems Science, vol. 43, no. 5, pp. 820-833, 2012.

[19] E. Tian, D. Yue, and C. Peng, "Brief Paper: reliable control for networked control systems with probabilistic sensors and actuators faults," IET Control Theory and Applications, vol. 4, no. 8, pp. 1478-1488, 2010.

[20] Z. Wang, D. W. C. Ho, Y. Liu, and X. Liu, "Robust $H_{\infty}$ control for a class of nonlinear discrete time-delay stochastic systems with missing measurements," Automatica, vol. 45, no. 3, pp. 684-691, 2009.

[21] H. Gao, T. Chen, and J. Lam, "A new delay system approach to network-based control," Automatica, vol. 44, no. 1, pp. 39-52, 2008. 
[22] H. Gao, W. Sun, and P. Shi, "Robust sampled-data $H_{\infty}$ control for vehicle active suspension systems," IEEE Transactions on Control Systems Technology, vol. 18, no. 1, pp. 238-245, 2010.

[23] W. Sun, H. Gao, and O. Kaynak, "Finite frequency $H_{\infty}$ control for vehicle active suspension systems," IEEE Transactions on Control Systems Technology, vol. 19, no. 2, pp. 416-422, 2011.

[24] M. Liu, D. W. C. Ho, and Y. Niu, "Stabilization of Markovian jump linear system over networks with random communication delay," Automatica, vol. 45, no. 2, pp. 416-421, 2009.

[25] L. Liu, Z. Han, and W. Li, " $H_{\infty}$ non-fragile observer-based sliding mode control for uncertain time-delay systems," Journal of the Franklin Institute, vol. 347, no. 2, pp. 567-576, 2010.

[26] M. Liu and G. Sun, "Observer-based sliding mode control for Itô stochastic time-delay systems with limited capacity channel," Journal of the Franklin Institute, vol. 349, no. 4, pp. 1602-1616, 2012. 


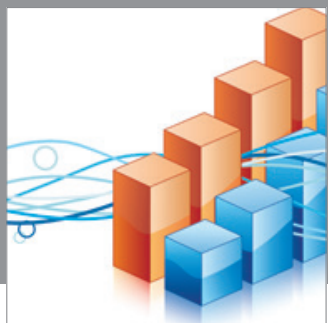

Advances in

Operations Research

mansans

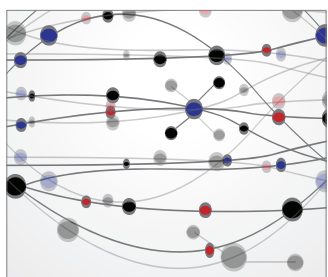

The Scientific World Journal
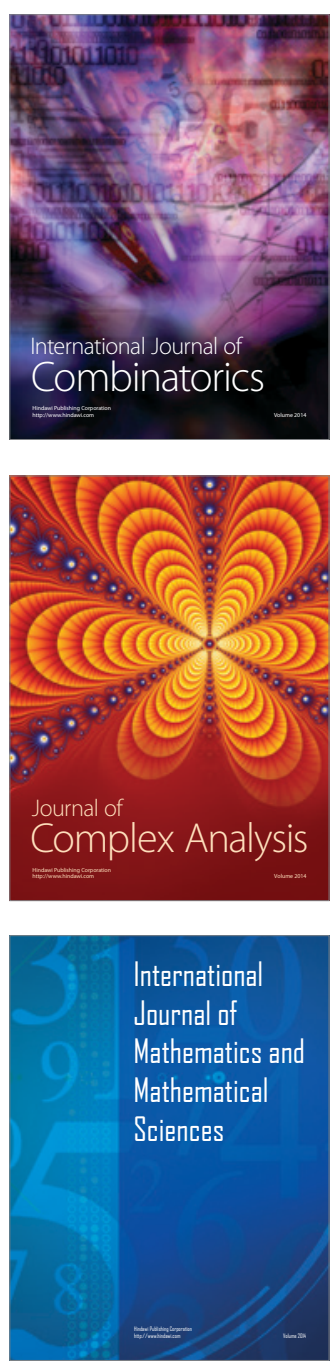
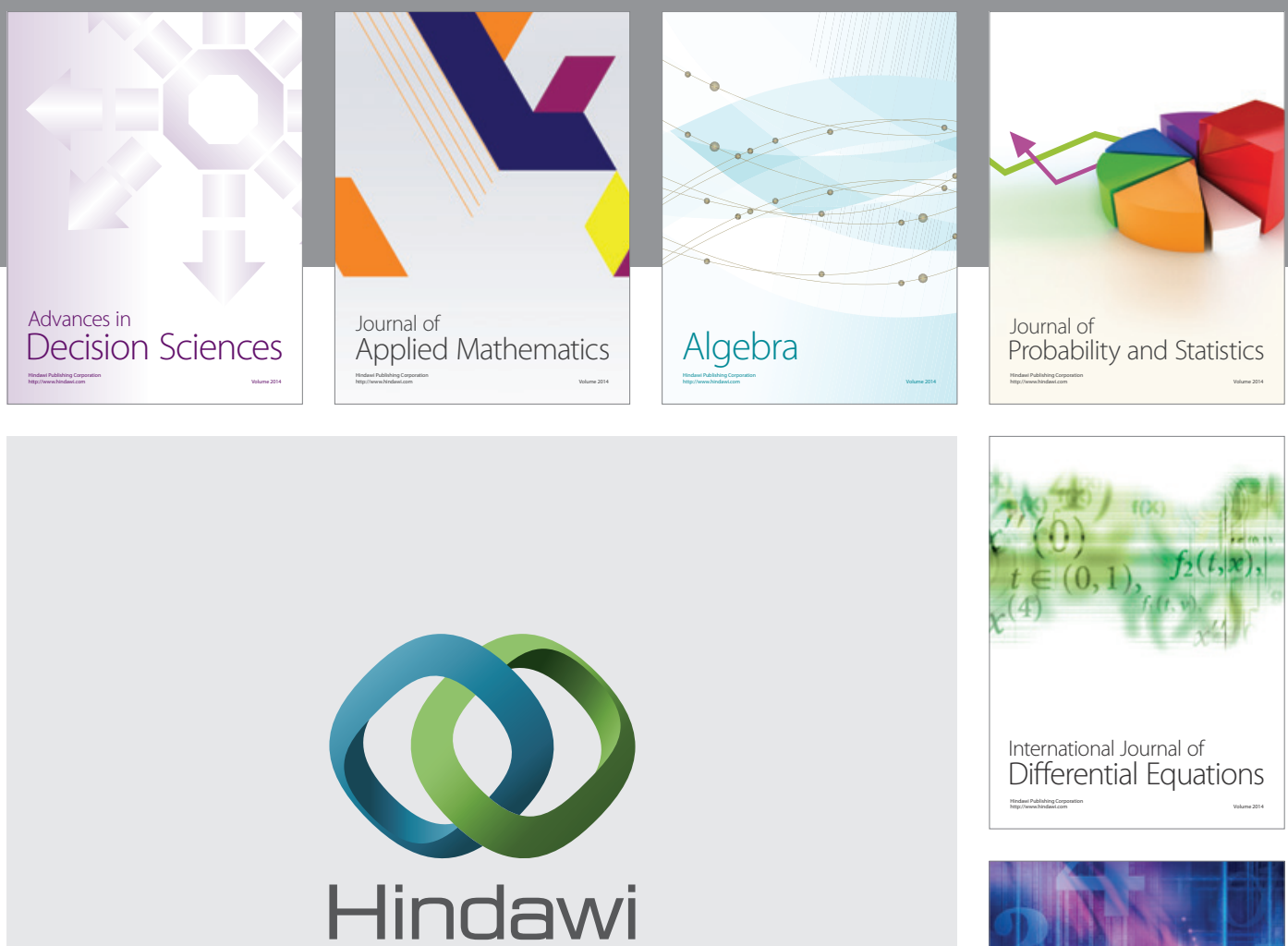

Submit your manuscripts at http://www.hindawi.com
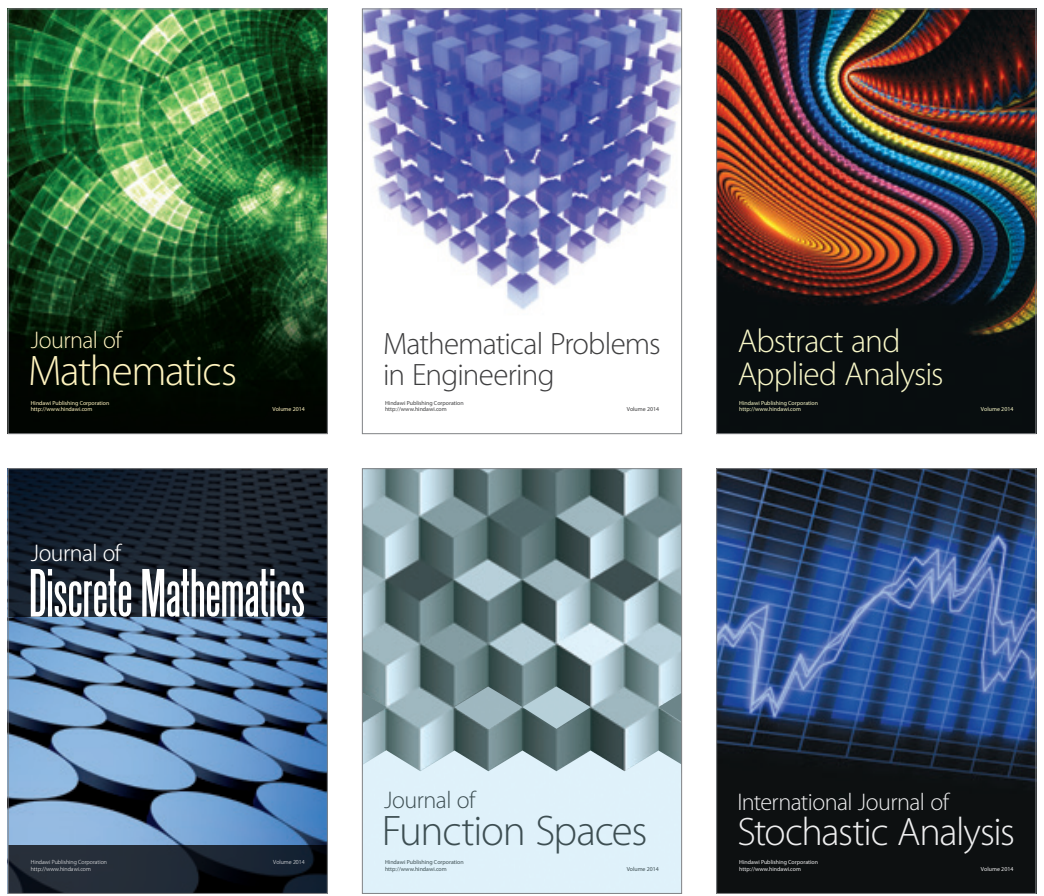

Journal of

Function Spaces

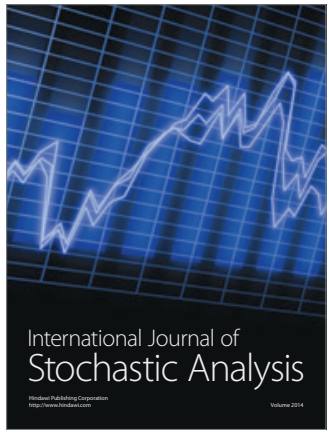

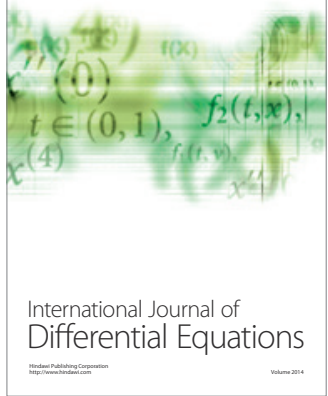
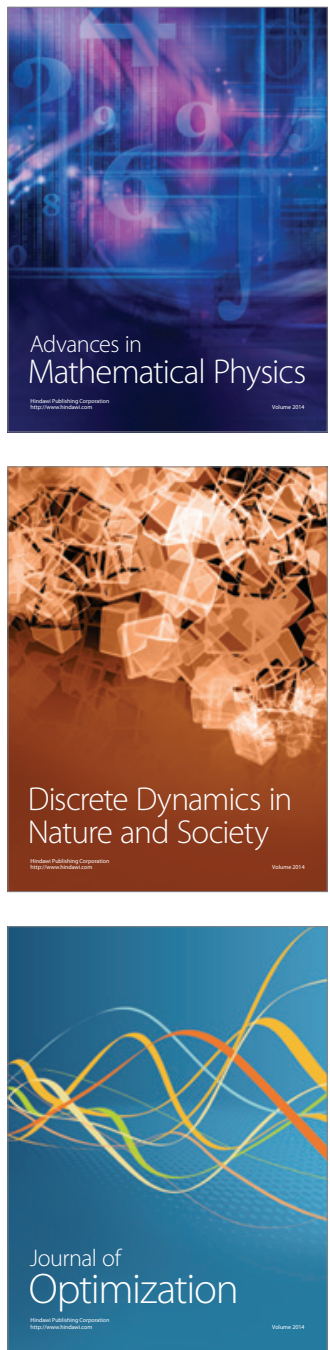